\title{
Retrospective Analysis of Clinical Laboratory Parameters, Therapeutics and Outcome in Patients Infected with SARS-CoV2
}

\author{
Sohini Sengupta ${ }^{1,2} \cdot$ Anil Handoo $^{2} \cdot$ Rajesh Pande $^{3} \cdot$ R. K. Kapoor ${ }^{1}$
}

Received: 28 June 2021 / Accepted: 23 September 2021 / Published online: 10 November 2021

(C) The Author(s), under exclusive licence to Association of Clinical Biochemists of India 2021

\begin{abstract}
Abstarct SARS-CoV-2 is the third coronavirus to have caused severe disease in humans in the last two decades, with approximately $5 \%$ of all patients and $20 \%$ of hospitalized patients experiencing severe symptoms, necessitating intensive care. The occurrence of Cytokine Storm has been implicated in the immune-pathogenesis of severe COVID-19. This is associated with cardiac injury, precipitated by cytokine mediated imbalance of coagulation and fibrinolysis, in the lung alveoli. In the absence of proven therapeutic agents, combinations of anti-viral drugs, immune-modulators and other adjunctive therapies have been tried in different clinical settings. A total of 128 confirmed cases of severe COVID-19 admitted to BLKMAX Super Speciality Hospital between 16th of June to 31 st of July, 2020 were included in this study. The correlation of age, gender, first value (on admission) of serum IL-6 and D-dimer, and impact of Tocilizumab and Remdesivir therapy on clinical outcome (28-day mortality), was evaluated in confirmed cases of severe COVID-19. The mortality rate was highest in the age group above 70 years. The incidence of death was significantly higher in males above 50 years, when age and gender were considered together. IL-6 and D-dimer levels $>70 \mathrm{pg} / \mathrm{mL}$ and $>$ $0.5 \mu \mathrm{g} \mathrm{FEU} / \mathrm{mL}$ respectively, were associated with poor outcome. $85.3 \%$ of patients treated with Remdesivir
\end{abstract}

Rajesh Pande

rajeshmaitree2000@gmail.com

1 Department of Clinical Biochemistry, BLK-MAX Super Speciality Hospital, Delhi, India

2 Department of Laboratory Services, BLK-MAX Super Speciality Hospital, Delhi, India

3 Department of Critical Care Medicine, BLK-MAX Super Speciality Hospital, Delhi, India showed clinical improvement. When Tocilizumab and Remdisivir were administered together, $44.0 \%$ of patients survived while $56 \%$ expired. $79.7 \%$ of patients survived while $20.3 \%$ expired when neither Tocilizumab nor Remdesivir was administered.

Keywords Cytokine Storm · IL-6 · D-dimer · Remdesivir Tocilizumab

\section{Introduction}

The Coronavirus Disease 2019 (COVID-19) has affected more than 179 million people worldwide with over 38 lakh confirmed deaths [1]. There are four different mechanisms by which the SARS CoV-2 virus may induce pathogenicity on entry into the host cell: direct cytotoxic effect, altered modulation of the RAAS (Renin- Angiotensin- Aldosterone System), endothelial cell damage with thromboembolism, and dysregulation of the immune system [2].

The immune response in the human body is mediated by cytokines which results in a protective well-controlled immune and inflammatory response. A particularly virulent viral infection may induce the secretion of extremely high levels of cytokines that induce secretion of more inflammatory mediators, resulting in a Cytokine Storm [3].

Available literature suggests that infection of pneumocytes by SARS-CoV-2 induces a local inflammatory response with secretion of cytokines like IL-1B, IFN- $\gamma$, IP10 , MCP-, TNF $\infty$, IL-4 and IL-10, amongst others [4]. The serum levels of IL- 2 and IL- 6 in patients with COVID19 have been found to correlate positively with the severity of COVID-19 [4, 5]. A cascade of destructive events is initiated, which ultimately results in acute lung injury or its more severe form ARDS (Acute respiratory distress 
syndrome), a major cause of mortality in COVID-19 [6]. The cytokine storm may also result in extra-pulmonary multiple-organ failure seen in some COVID-19 patients with or without respiratory failure [2]. Evidence suggests that IL-6 is an independent prognostic marker and has generated interest in its estimation and further evaluation as an outcome predictor [7].

Patients with severe COVID-19 infection have been reported to suffer from cardiac injury and coagulation disorders $[8,9]$. An imbalance of coagulation and fibrinolysis in the alveoli by inflammatory cytokines could activate the fibrinolytic system and result in increased D-dimer levels [10]. Abnormal coagulation functions with elevated D-dimer levels have been found to be more common in deceased patients of COVID-19, with a higher incidence of death when the value was greater than $1 \mu \mathrm{g} / \mathrm{ml}$ $[11,12]$.

With this background, and in the absence of any significant published data from India on the correlation between the serum level of IL-6, D-dimer and final outcome of patients infected with SARS-CoV-2, we have analyzed the probable impact of age, gender and varying levels of these inflammatory biomarkers on laboratory confirmed cases of COVID-19. The role of the IL-6 antagonist (Tocilizumab) and anti-viral therapy (Remdesivir) on the prognosis of patients was also reviewed.

\section{Materials and Methods}

This single-center, retrospective observational study was carried out after approval by the institutional Research Ethics Committee, in BLK Super Specialty Hospital, Delhi, which is a designated tertiary care referral hospital for treatment of COVID-19 patients. The primary objective of the study was to evaluate the correlation of the first value (on admission) of serum IL-6 and D-dimer with the outcome (28-day mortality) in confirmed cases of severe COVID-19. The secondary objectives included effect of age and gender and impact of Tocilizumab and Remdesivir therapy on outcome in these patients.

A total of 128 confirmed cases of severe COVID-19, above 18 years of age admitted in the COVID ICU between the 16th of June 2020, and 31st of July 2020 were included in this study. All patients were confirmed by SARS-CoV-2 nucleic acid RT-PCR (Positive) or Rapid Antigen Testing (Positive) using specimens derived from nasopharyngeal and/or oropharyngeal swabs, prior to or during hospitalization. All patients were monitored via the hospital information system, and clinical data were collected until 31st of July 2020, the last date of follow-up.

Data on demographic characteristics and laboratory tests were collected from medical records. Time from disease onset to hospitalization, duration of hospitalization was also recorded. Fasting venous plasma samples were collected within $6 \mathrm{~h}$ of admission on day 1 for measurement of IL-6 and D-dimer and were analyzed within 2 h. IL- 6 was measured by electrochemiluminescence immunoassay (Kit: Elecsys IL-6) on Cobas e-411® immunoassay analyzer. D-dimer was measured by particle-enhanced immunoturbidimetric assay (Kit: D-DI2) on Cobas c-501® analyzers.

\section{Definition of Severe Covid-19}

Severe COVID-19 pneumonia was defined as severe pneumonia in adolescent or adults with clinical signs of pneumonia plus one of the following: respiratory rate $>30$ breaths/min, severe respiratory distress, breaths/min, severe respiratory distress, $\mathrm{SpO} 2<90 \%$ on room air [13]. The definitions for ARDS, sepsis and septic shock were adopted from this guideline document.

Standard therapy: All severe COVID-19 patients were given prophylactic antibiotics, Remdesivir 200-mg on day one, followed by $100 \mathrm{mg}$ for next four days in patients who were improving and for a total of 10 day in patients who had a worsening hypoxemia and/or continued to be on high ventilatory support, dexamethasone 6-mg OD for 10 days or until discharge from ICU in patients who were in oxygen therapy/NIV or invasive ventilation, and prophylactic subcutaneous enoxaparin or deltaparin which was escalated to therapeutic dosing if D-dimer was more than 3 time normal. Tocilizumab was administered in a dose of $8 \mathrm{mg} /$ $\mathrm{kg} \mathrm{IV}$, if IL-6 levels were raised more than 10 times normal $(>70)$ and was repeated up to three times if the patient continued to be critical and the inflammatory markers remained persistently high or showed an increasing trend. In addition, vitamin $\mathrm{C}(1 \mathrm{~g} / \mathrm{day})$ and zinc were also given besides other supportive treatment. Convalescent plasma in two aliquots of $200 \mathrm{ml}$ each on two successive days was given to all patients unless the D-Dimer was $>5$ times normal.

Statistical analysis: Statistical package for the social science system version SPSS 17.0 (Chicago, IL, USA) was used for statistical analysis. Results were expressed as mean $\pm \mathrm{SD}$, median or numbers and percentages. Differences between groups were assessed with Chi-square for categorical variables. Fishers exact test was used if expected frequencies were $<5$. A ' $p$ ' value of $<0.05$ was taken as significant.

\section{Results}

A total of 128 patients with confirmed SARS CoV-2 infection classified as severe were included in this study. The mortality rate was found to be significantly higher with 
increasing age. The maximum mortality occurred in the age group above 70 years. The incidence of death was significantly higher in males above 50 years of age when both age and gender were considered together. $(p<0.01)$ (Table 1, Fig. 1) The cut-off values for IL-6 were $<7 \mathrm{pg} /$ $\mathrm{mL}$ (normal), $7-70 \mathrm{pg} / \mathrm{mL}$ (high) and more than $70 \mathrm{pg} / \mathrm{mL}$ (very high) according to the clinical practice adopted in the hospital. The mortality rate correlated well with increasing values of IL- 6 , with the maximum incidence of deaths occurring at an IL-6 value $>70 \mathrm{pg} / \mathrm{mL} \quad(\mathrm{p}<0.001)$ (Table 2, Fig. 2b) The Biological Reference Interval for D-dimer is less than $0.5 \mu \mathrm{g}$ FEU/mL, values above which were considered as high. The higher levels of D-dimer, above $0.5 \mu \mathrm{g}$ FEU/mL were found to be strongly associated with increased risk of poor outcome $(p<0.01)$ (Table 3 Fig. 2b). While $80 \%$ of patients who were administered Tocilizumab recovered completely, $85.3 \%$ improved when treated with Remdesivir alone. However, $65 \%$ of patients on treatment with both Tocilizumab and Remdesivir expired. (Table 4, Fig. 3a) A borderline significant association was found between mortality rate and higher levels of IL-6 when Tocilizumab was administered. (Table 5, Fig. 3b).

\section{Discussion}

Our study shows that the probability of death increases with age in patients of severe COVID-19, with the case fatalities being highest in the age group of over seventy years. This correlates with available literature which mention that older COVID-19 patients are at an increased risk of death due to the disease [14-16]. Our analysis also shows a significantly higher mortality in males above fifty

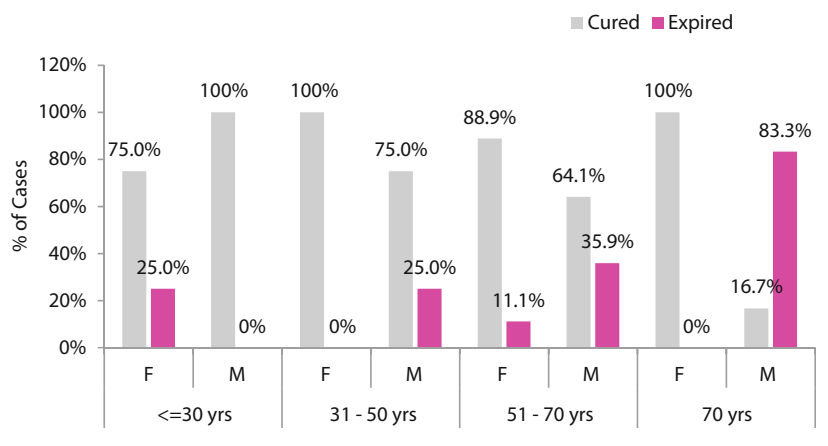

Fig. 1 Correlation of outcome of patients with gender and age

Table 2 The mortality rate is significantly associated with higher IL6 value $(p$ value $<0.001)$

\begin{tabular}{|c|c|c|c|c|c|c|}
\hline \multirow[t]{3}{*}{ IL-6 } & \multirow[t]{3}{*}{ Total } & \multicolumn{4}{|l|}{ Outcome } & \multirow[t]{3}{*}{$p$ value } \\
\hline & & \multicolumn{2}{|l|}{ Cured } & \multicolumn{2}{|l|}{ Expired } & \\
\hline & & Frequency & $\%$ & Frequency & $\%$ & \\
\hline$<7$ & 41 & 38 & 92.7 & 3 & 7.3 & $<0.001$ \\
\hline $7-70$ & 52 & 43 & 82.7 & 9 & 17.3 & \\
\hline$>70$ & 35 & 14 & 40.0 & 21 & 60.0 & \\
\hline Total & 128 & 95 & 74.2 & 33 & 25.8 & \\
\hline
\end{tabular}

years of age, when compared to females, which corelates with global sex-aggregated data [16]. Our study correlates well with published data showing a significant association between higher values of IL-6 and mortality. 92.7\% of patients, who had IL-6 less than $7 \mathrm{pg} / \mathrm{mL}$ improved clinically and were discharged. $82.7 \%$ of infected patients with IL-6 values between $7-70 \mathrm{pg} / \mathrm{mL}$, improved. However, $60 \%$ of patients with IL-6 value above $70 \mathrm{pg} / \mathrm{mL}$ expired.
Table 1 The mortality rate is significantly higher in males in age group of more than 50 years. $(p$ value $<0.01)$

\begin{tabular}{|c|c|c|c|c|c|c|c|}
\hline \multirow[t]{3}{*}{ Age Groups } & \multirow[t]{3}{*}{ Gender } & \multirow[t]{3}{*}{ Total } & \multicolumn{4}{|l|}{ Outcome } & \multirow[t]{3}{*}{$p$ value } \\
\hline & & & \multicolumn{2}{|l|}{ Cured } & \multicolumn{2}{|l|}{ Expired } & \\
\hline & & & Frequency & $\%$ & Frequency & $\%$ & \\
\hline \multirow{3}{*}{$<=30$ years } & $\mathrm{F}$ & 4 & 3 & 75.0 & 1 & 25.0 & 0.308 \\
\hline & M & 9 & 9 & 100.0 & 0 & 0.0 & \\
\hline & Total & 13 & 12 & 92.3 & 1 & 7.7 & \\
\hline \multirow[t]{3}{*}{$31-50$ years } & $\mathrm{F}$ & 12 & 12 & 100.0 & 0 & 0.0 & 0.130 \\
\hline & M & 20 & 15 & 75.0 & 5 & 25.0 & \\
\hline & Total & 32 & 27 & 84.4 & 5 & 15.6 & \\
\hline \multirow[t]{3}{*}{$51-70$ years } & $\mathrm{F}$ & 27 & 24 & 88.9 & 3 & 11.1 & 0.043 \\
\hline & M & 39 & 25 & 64.1 & 14 & 35.9 & \\
\hline & Total & 66 & 49 & 74.2 & 17 & 25.8 & \\
\hline \multirow[t]{3}{*}{70 years } & $\mathrm{F}$ & 5 & 5 & 100.0 & 0 & 0.0 & 0.003 \\
\hline & M & 12 & 2 & 16.7 & 10 & 83.3 & \\
\hline & Total & 17 & 7 & 41.2 & 10 & 58.8 & \\
\hline
\end{tabular}




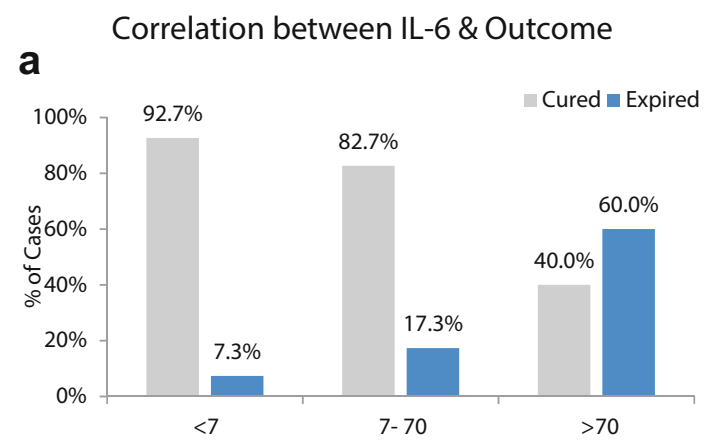

b

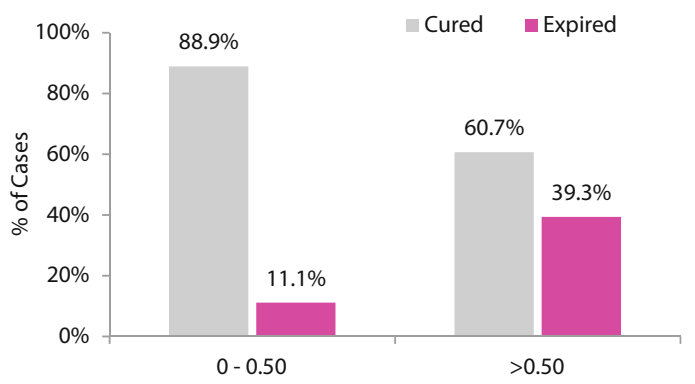

Fig. 2 a Correlation of IL-6 levels with outcome of patients. b Correlation of D-dimer levels with outcome of patients

Table 3 The mortality rate is significantly associated with higher D dimer levels. $(p$ value $<0.01)$

\begin{tabular}{|c|c|c|c|c|c|c|}
\hline \multirow[t]{3}{*}{ D-dimer } & \multirow[t]{3}{*}{ Total } & \multicolumn{4}{|l|}{ Outcome } & \multirow[t]{3}{*}{$p$ value } \\
\hline & & \multicolumn{2}{|l|}{ Cured } & \multicolumn{2}{|l|}{ Expired } & \\
\hline & & Frequency & $\%$ & Frequency & $\%$ & \\
\hline $0-0.50$ & 27 & 24 & 88.9 & 3 & 11.1 & 0.011 \\
\hline$>0.50$ & 61 & 37 & 60.7 & 24 & 39.3 & \\
\hline Total & 88 & 61 & 69.3 & 27 & 30.7 & \\
\hline
\end{tabular}

Our findings are similar to the results of a multicenter retrospective study which reported significantly higher levels of IL-6 in non-survivors compared to resolving cases [4]. In a similar study by Gao et al., levels of IL-6 were reported to be significantly higher in severe disease compared to mild [5]. An increasing mean IL-6 on admission was found to be associated with an increased likelihood of mortality and a cut-off value greater than $55 \mathrm{pg} / \mathrm{mL}$ has been suggested for identifying patients at high risk of severe COVID-19 [17]. Another study identified values more than $80 \mathrm{pg} / \mathrm{mL}$ for identifying patients at high risk of mortality [18]. This was consistent with the proposed concept of "cytokine storm" which observed that inflammatory factors played a crucial role in the progression of COVID-19 from mild to severe disease [19]. In another study, all deaths exhibited extremely high IL-6 value, suggesting its role in indicating a poor prognosis for COVID-19 patients [20]. Our study also observed that IL-6 may be used as a predictive biomarker for the evaluation of severity of infection, prediction of prognosis and response to treatment in patients with severe COVID 19. IL-6 at the time of hospitalization was found to be a strong and independent predictor of patient survival in severe COVID$19(\mathrm{p}<0.001)$.

The mortality rate was found to significantly high in patients with a D-dimer value $>0.5 \mu \mathrm{g} \mathrm{FEU} / \mathrm{mL}$. An increased D-dimer level in $36 \%$ of patients infected with COVID-19 was shown in a previous study [9]. Wuhan hospitals reported a mortality of $28 \%$, which was associated with an elevated D-dimer $>1.0 \mu \mathrm{g} / \mathrm{mL}$ on admission [12]. Our study also correlates with an analysis which showed that markedly elevated D-dimer and FDP are commonly seen in deaths due to severe novel coronavirus pneumonia [11]. Another report showed that higher IL-6 levels correlated with increased fibrinogen levels, demonstrating a link between inflammation and pro-coagulant changes [8]. However, our study did not find any significant association between mortality rate and higher D-dimer levels at different cut-offs for IL-6.

Published preclinical data regarding efficacy of IL-6 inhibitors against SARS-CoV2 is not readily available. A viable option is the targeting of the expression of IL-6 with Tocilizumab, a monoclonal antibody against IL-6 receptor. Considering the proven role of cytokine dys-regulation in causing hyper-inflammation in the lungs (IL-6 being a key driver) particularly in seriously ill COVID-19 patients, selective cytokine blockade with Tocilizumab has been tested in some hospitals with varying results [21]. The antiviral drug Remdesivir (GS-5734), a nucleoside analogue prodrug, has also shown inhibitory effects on SARS-
Table 4 Correlation of outcome with administration of RMD, both TCZ and RMD, no use of TCZ and RMD

\begin{tabular}{|c|c|c|c|c|c|}
\hline & \multirow[t]{3}{*}{ Total patients } & \multicolumn{4}{|l|}{ Outcome } \\
\hline & & \multicolumn{2}{|l|}{ Cured } & \multicolumn{2}{|l|}{ Expired } \\
\hline & & Frequency & $\%$ & Frequency & $\%$ \\
\hline No TCZ\& No RMD & 69 & 55 & 79.7 & 14 & 20.3 \\
\hline RMD & 34 & 29 & 85.3 & 5 & 14.7 \\
\hline TCZ and RMD & 25 & 11 & 44.0 & 14 & 56.0 \\
\hline
\end{tabular}


a $\quad$ Cured $\quad$ Expired
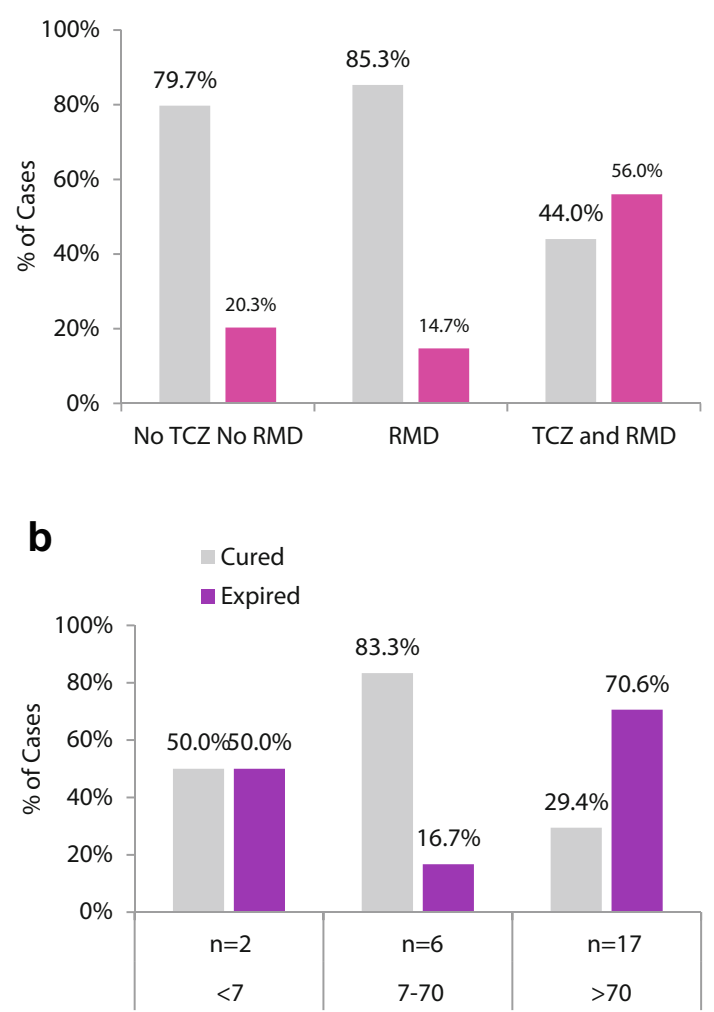

Fig. 3 a Correlation of outcome with administration of RMD, both TCZ and RMD, no use of TCZ and RMD b Correlation of outcome with levels of IL-6 when Tocilizumab was administered

Table 5 Correlation between mortality rates and IL- 6 values when TCZ is administered

\begin{tabular}{|c|c|c|c|c|c|c|}
\hline \multirow[t]{3}{*}{ IL-6 } & \multirow[t]{3}{*}{ TCZ given } & \multicolumn{4}{|l|}{ Outcome } & \multirow[t]{3}{*}{$p$ value } \\
\hline & & \multicolumn{2}{|l|}{ Cured } & \multicolumn{2}{|l|}{ Expired } & \\
\hline & & Frequency & $\%$ & Frequency & $\%$ & \\
\hline$<7$ & $\mathrm{n}=2$ & 1 & 50.0 & 1 & 50.0 & 0.052 \\
\hline $7-70$ & $\mathrm{n}=6$ & 5 & 83.3 & 1 & 16.7 & \\
\hline$>70$ & $\mathrm{n}=17$ & 5 & 29.4 & 12 & 70.6 & \\
\hline
\end{tabular}

CoV-2 in vitro, and has therefore been tested in patients of severe COVID-19 in some countries [22]. The National Health Commission of China (NHC) included Tocilizumab in its 7th updated diagnosis and treatment plan for COVID19 [23]. They are also mentioned in the NIH treatment guidelines and the WHO interim guidance, both of which cite insufficient clinical data to recommend either for or against the use of these drugs, as is the guideline for other drugs [24, 25]. Evidence of efficacy in COVID-19 is available for Tocilizumab through small studies. One study in severe COVID-19 patients showed successful outcomes in 21 patients, with most receiving only one dose of Tocilizumab $400 \mathrm{mg}$ along with standard of care. It showed improved clinical outcomes in $91 \%$ of patients and successful discharge with a mean of 15.5 days [26]. A retrospective observational study in 15 COVID-19 patients reported a good response in those treated with Tocilizumab and recommend repeated doses in critically ill COVID-19 patients [27]. Some other studies involving the off-label use of Tocilizumab have also shown the potential efficacy of this drug in the treatment of COVID- 19 [28]. No specific antiviral drug has been proven effective for treatment of patients with COVID-19. However, in a cohort of patients hospitalized for severe COVID-19 and treated with compassionate-use Remdesivir, clinical improvement was observed in 36 of 53 patients (68\%) [29]. One randomized control trial found that intravenous Remdesivir did not significantly improve the time to clinical improvement, mortality, or time to clearance of virus in patients with serious COVID-19 compared with placebo [22]. In our study, $85.3 \%$ of patients treated with Remdesivir alone, (29 out of 34) showed clinical improvement. When Tocilizumab and Remdisivir were administered together, $44.0 \%$ of patients survived while $56 \%$ expired. We also found a borderline significant association with mortality rate when Tocilizumab was administered at higher levels of IL-6 (>70 pg/mL). $79.7 \%$ of patients survived while $20.3 \%$ expired when neither Tocilizumab nor Remdesivir were administered. According to the WHO Solidarity Trial results, Remdesivir amongst others was found to have little or no effect on hospitalized COVID-19, as indicated by overall mortality, initiation of ventilation and duration of hospital stay [30]. The Adaptive Covid-19 Treatment Trial showed that while Remdesivir led to a shorter median time to recovery and reduced the time to hospital discharge, it did not show a reduction in mortality [31]. In a case reported on two patients where one was treated with Tocilizumab alone, and the other with Tocilizumab and Remdisivir, both showed a similar outcome [32]. The higher mortality rate associated with the use of both these drugs may be attributed to the severity of infection and an exaggerated immune response in these cases.

\section{Conclusion}

Our study concluded that men above fifty years of age were particularly vulnerable to a poor outcome in severe COVID-19 disease. Higher levels of IL-6 and D-dimer were found to correlate well with increased mortality. Both these biomarkers may therefore be estimated on admission, and serially thereafter, to guide the clinicians in recognizing patients with severe COVID-19 early in the disease course, and monitor prognosis. The antiviral drug 
Remdesivir improved clinical outcome in the study population. The mortality rate was however higher when Remdisivir and Tocilizumab were administered together.

\section{Limitations}

The small sample size was a limiting factor of this study. The frequency and total number of samples collected for estimation of IL-6 and D-dimer was not uniform. It is important that researchers develop a scoring system including estimation of IL-6 and D-dimer at defined intervals to assist clinicians in early recognition of patients at risk for developing severe disease. The usefulness of Tocilizumab and Remdesivir in COVID-19 in the Indian scenario also needs to be explored on a larger sample size.

Authors' Contributions All authors whose names appear on the submission made substantial contributions to the conception or design of the work; or the acquisition, analysis, or interpretation of data.

Funding No funds, grants, or other support was received.

\section{Declarations}

Conflict of interest The authors have no relevant financial or nonfinancial interests to disclose.

Ethics Approval Ethical approval was waived by the Institutional Ethics Committee of BLK-MAX Super Speciality Hospital, Delhi, in view of the retrospective nature of the study and all the procedures being performed were part of the routine care.

\section{References}

1. WHO Coronavirus Disease (COVID-19) Dashboard. https:// covid19.who.int/. Accessed on 28.6.2021.

2. Gubernatorova EO, Gorshkova EA, Polinovaa AI, Drutskaya MS. IL-6: Relevance for immunopathology of SARS-CoV-2. Cytokine Growth Factor Rev. 2020;53:13-24.

3. Kuby Immunology (2013) Receptors and Signaling: Cytokines and Chemokines. 7th Edition. P 137. W H Freeman and Company. New York.

4. Ruan Q, Yang K, Wang W, Jiang L, Song J. Clinical predictors of mortality due to COVID-19 based on an analysis of data of 150 patients from Wuhan, China. Intensive Care Med. 2020;46:846-8. https://doi.org/10.1007/s00134-020-06028-z.

5. Gao Y, Li T, Han M, Li X, Wu D, Xu Y, et al. Diagnostic utility of clinical laboratory data determinations for patients with the severe COVID-19. J Med Virol. 2020;92:791-6. https://doi.org/ 10.1002/jmv.25770.

6. Ragab D, Salah Eldin H, Taeimah M, Khattab R, Salem R. The COVID-19 Cytokine storm; what we know so far. Front Immunol. 2020;11:1446. https://doi.org/10.3389/fimmu.2020.01446.

7. Ye Q, Wang B, Mao J. The pathogenesis and treatment of the 'Cytokine Storm' in COVID-19. J Infect. 2020;80:607-13.

8. Ranucci M, Ballotta A, Di Dedda U, et al. The procoagulant pattern of patients with COVID-19 acute respiratory distress syndrome. J Thromb Haemost. 2020. https://doi.org/10.1111/jth. 14854.

9. Connors J, Levy J. COVID-19 and its implications for thrombosis and anticoagulation. Blood. 2020;135:2033-40.

10. Tang N, Bai H, Chen X, Gong J, Li D, Sun Z. Anticoagulant treatment is associated with decreased mortality in severe coronavirus disease 2019 patients with coagulopathy. J Thromb Haemost. 2020;18(5):1094-9. https://doi.org/10.1111/jth.14817.

11. Tang N, Li D, Wang X. Abnormal coagulation parameters are associated with poor prognosis in patients with novel coronavirus pneumonia. J Thromb Haemost. 2020;18(4):844-7. https://doi. org/10.1111/jth.14768.

12. Zhou F, Yu T, Du R. Clinical course and risk factors for mortality of adult inpatients with COVID-19 in Wuhan, China: a retrospective cohort study. Lancet. 2020;395(10229):1054-62. https:// doi.org/10.1016/s0140-6736(20)30566-3.

13. Clinical Guidance for Management of Adult COVID-19 Patients. https://www.icmr.gov.in/pdf/covid/techdoc/COVID_Manage ment_Algorithm_17052021.pdf. (Accessed on 25.10. 2020)

14. Wu C, Chen X, Cai Y, et al. Risk factors associated with acute respiratory distress syndrome and death in patients with coronavirus disease 2019 Pneumonia in Wuhan, China. JAMA Intern Med. 2020;180(7):934-43. https://doi.org/10.1001/jamain ternmed.2020.0994.

15. Yang $\mathrm{X}, \mathrm{Yu} \mathrm{Y}, \mathrm{Xu} \mathrm{J}$, et al. Clinical course and outcomes of critically ill patients with SARS-CoV-2 pneumonia in Wuhan, China: a single-centered, retrospective, observational study. Lancet Respir Med. 2020;8:475-81. https://doi.org/10.1016/ S2213-2600(20)30079-5.

16. Sex, gender and Covid-19. 2020. Global Health 5050. https:// globalhealth5050.org/covid19/ (Date accessed: 18.11.2020).

17. Aziz M, Fatima R, Assaly R. Elevated interleukin-6 and severe COVID-19: a meta-analysis. J Med Virol. 2020. https://doi.org/ 10.1002/jmv.25948.

18. Chen X, Zhao B, Qu Y, Chen Y, Xiong J, Feng Y, Men D, Huang Q, Liu Y, Yang B, et al. Detectable serum SARS-CoV-2 viral load (RNAaemia) is closely associated with drastically elevated interleukin 6 (IL-6) level in critically ill COVID-19 patients. Clin Infect Dis. 2020. https://doi.org/10.1093/cid/ciaa449.

19. Liu F, Li L, Xu M, Wu J, Luo D, Zhu Y, Li B, Song X, Zhou X. Prognostic value of interleukin-6, C-reactive protein, and procalcitonin in patients with COVID-19. J Clin Virol. 2020;127:104370.

20. Chen T, Wu D, Chen H, et al. Clinical characteristics of 113 deceased patients with coronavirus disease 2019: retrospective study. BMJ. 2020;368:m1091.

21. Xu X, Han M, Li T, Sun W, Wang D, Fu B, et al. Effective treatment of severe COVID-19 patients with tocilizumab. Proc Natl Acad Sci. 2020. https://doi.org/10.1073/pnas.2005615117.

22. Wang Y, Zhang D, Du G, Du R, Zhao J, Jin Y, et al. Remdesivir in adults with severe COVID-19: a randomised, double-blind, placebo-controlled, multicentre trial. Lancet. 2020;395(10236):1569-78. https://doi.org/10.1016/S01406736(20)31022-9.

23. National Health Commission. Diagnosis and treatment protocol for novel coronavirus pneumonia (Trial Version 7). Chin Med J (Engl). 2020;133(9):1087-95.

24. World Health Organization (WHO). Clinical Management of COVID-19. Interim Guidance. 27 May. 2020.

25. National Institutes of Health. Coronavirus disease 2019 (COVID19) treatment guidelines. 2020. Available at: https://www.cov id19treatmentguidelines.nih.gov/. Accessed on 18.11.20

26. Cennimo DJ, Bergman SJ. Coronavirus Disease 2019 (COVID19) Treatment \& Management. Updated. 2020. Available at: http://emedicine.medscape.com/article/2500114-treatment. Accessed on 18.11.20. 
27. Treatment options. In: Joseph T, Moslehi MA, editors. International Pulmonologist's Consensus On COVID-19, 2nd edn. Available at: https://www.unah.edu.hn/dmsdocument/9674-con senso-internacional-de-neumologos-sobre-covid-19-versioningles. Accessed on 18.11.20

28. Morena V, Milazzo L, Oreni L, Bestetti G, Fossali T, Bassoli C, et al. Offlabel use of tocilizumab for the treatment of SARS-CoV2 pneumonia in Milan. Italy Eur J Intern Med. 2020;76:36-42. https://doi.org/10.1016/j.ejim.2020.05.011.

29. Grein J, Ohmagari N, Shin D, Diaz G, Asperges E, Castagna A, et al. Compassionate use of remdesivir for patients with severe Covid-19. N Engl J Med. 2020;382(24):2327-36.

30. Solidarity Therapeutics Trial produces conclusive evidence on the effectiveness of repurposed drugs for COVID-19 in record time. https://www.who.int/news/item/15-10-2020-solidarity-ther apeutics-trial-produces-conclusive-evidence-on-the-effective ness-of-repurposed-drugs-for-covid-19-in-record-time. Accessed on 20.11.20.

31. Beigel JH, Tomashek KM, Dodd LE, et al. Remdesivir for the treatment of Covid-19-final report. $N$ Engl J Med. 2020;383:1813-26.

32. Akinosoglou K, et al. Remdesivir and tocilizumab: mix or match. J Med Virol. 2020. https://doi.org/10.1002/jmv.26117.

Publisher's Note Springer Nature remains neutral with regard to jurisdictional claims in published maps and institutional affiliations. 